\title{
The effects of sevoflurane with propofol and remifentanil on tracheal intubation conditions without neuromuscular blocking agents
}

\author{
Wook Jong Kim, Seong Soo Choi, Doo Hwan Kim, Hye Jeong Seo, Eun Ha Suk, Seung Woo Ku, and \\ Pyung Hwan Park \\ Department of Anesthesiology and Pain Medicine, Asan Medical Center, University of Ulsan College of Medicine, Seoul, Korea
}

Background: Propofol and remifentanil are used for tracheal intubation in the absence of neuromuscular blocking agents. We hypothesized that the addition of sevoflurane to propofol and remifentanil would improve intubation conditions and provide hemodynamic stability.

Methods: Seventy-six patients scheduled for elective surgery were randomly allocated to be ventilated with either $4 \%$ (group I) or $7 \%$ sevoflurane (group II) after propofol injection ( $2 \mathrm{mg} / \mathrm{kg}$ ). All patients received remifentanil $(1 \mu \mathrm{g} / \mathrm{kg})$ 30 seconds after administration of propofol. Ninety seconds after remifentanil was given, laryngoscopy and tracheal intubation were performed. Intubation conditions and hemodynamic changes were evaluated.

Results: The overall incidence of clinically acceptable intubation conditions was significantly higher in group II (92\%) than group I (58\%) $(\mathrm{P}=0.001)$. Scores for vocal cord position, coughing, and limb movement were significantly better in group II $(\mathrm{P}<0.05)$. Mean blood pressure remained significantly lower than the pre-induction level throughout the investigation in both groups $(\mathrm{P}<0.001)$, but there was no incidence of bradycardia or hypotension requiring treatment.

Conclusions: Tracheal intubation without neuromuscular blocking agents can be achieved safely and reliably by adding 7\% sevoflurane to propofol (2 mg/kg) and remifentanil (1 $\mu \mathrm{g} / \mathrm{kg})$. (Korean J Anesthesiol 2010; 59: 87-91)

Key Words: Intubation, Propofol, Remifentanil, Sevoflurane.

Received: February 10, 2010. Revised: 1st, March 1, 2010; 2nd, March 30, 2010. Accepted: May 10, 2010.

Corresponding author: Eun Ha Suk, M.D., Department of Anesthesiology and Pain Medicine, Asan Medical Center, University of Ulsan School of Medicine, 388-1, Pungnap-dong, Songpa-gu, Seoul 138-040, Korea. Tel: 82-2-3010-3868, Fax: 82-2-3010-3690, E-mail: eusuk@medigate.net (c) This is an open-access article distributed under the terms of the Creative Commons Attribution Non-Commercial License (http:// creativecommons.org/licenses/by-nc/3.0/), which permits unrestricted non-commercial use, distribution, and reproduction in any medium, provided the original work is properly cited. 


\section{Introduction}

Although tracheal intubation is facilitated by muscle relaxants, neuromuscular blocking agents may be contraindicated (e.g., in patients with known allergic reactions or myopathies) or associated with side effects such as prolonged paralysis, awareness, or complications of residual neuromuscular blockade. This has led to intubation without neuromuscular blocking agents (IWNA). Ambulatory anesthesia requires a rapid, safe recovery of spontaneous breathing. IWNA is also useful for short duration anesthesia when intubation is necessary but neuromuscular blockage is not.

Optimal combinations of hypnotic agents and opioids should provide satisfactory intubation conditions without neuromuscular blockade. Propofol produces superior relaxation of the jaw $[1,2]$ and suppression of airway reflexes compared with other intravenous induction agents $[3,4]$, whereas remifentanil is an ultra-short acting opioid with rapid onset and strong potency, making it ideal for short noxious stimulation such as intubation. Thus, various combinations of propofol and remifentanil have been proposed for IWNA [5-10]. Sevoflurane, a nonirritating inhaled anesthetic agent with low blood gas solubility, has also been used for IWNA, either alone or combined with remifentanil [11-14]. In the absence of neuromuscular blocking agents, however, a relatively large dose of propofol or remifentanil and a high concentration of sevoflurane are required for longer durations, which may lead to hemodynamic instability [8-11].

We hypothesized that the addition of sevoflurane to propofol and remifentanil would allow the use of lower doses of propofol and remifentanil as well as reduce the incidence of hemodynamic instability. To date, there have been no studies on intubation conditions using balanced administration of propofol, remifentanil, and sevoflurane. We therefore investigated the effects of sevoflurane combined with propofol $(2 \mathrm{mg} / \mathrm{kg})$ and remifentanil $(1 \mu \mathrm{g} / \mathrm{kg})$ on intubation conditions and hemodynamic responses in the absence of neuromuscular blocking agents.

\section{Materials and Methods}

This study was approved by our institutional review board and informed written consent was obtained from patients. We studied 76 American Society of Anesthesiologist Physical Status I patients, aged 16-60 years, undergoing elective surgery under general anesthesia requiring tracheal intubation. Exclusion criteria included a history of drug or alcohol abuse, upper gastrointestinal reflux, cardiovascular disease or reactive airway diseases, including smoking, body mass index 30 or more, allergies to any of the study drugs, administration of sedative or narcotic drugs in the previous $24 \mathrm{~h}$, renal or hepatic impairment, or a Mallampati classification of airway anatomy above class II.

Automated non-invasive arterial pressure (NIBP), electrocardiogram, pulse oximetry $\left(\mathrm{SpO}_{2}\right)$, and end tidal carbon dioxide concentrations $\left(\mathrm{ETCO}_{2}\right)$ were monitored in the anesthetic room. All patients were prehydrated with balanced salt solution $(5 \mathrm{ml} /$ $\mathrm{kg})$. Midazolam $(0.03 \mathrm{mg} / \mathrm{kg})$ and glycopyrrolate $(0.2 \mathrm{mg})$ were administered intravenously (IV) approximately 5 min before anesthesia.

Patients were allocated randomly to receive $4 \%$ (group I) or $7 \%$ sevoflurane (group II), based on the results of a small pilot study, with assignment decided using sealed envelopes. After 3 min of pre-oxygenation, anesthesia was induced with propofol ( $2 \mathrm{mg} / \mathrm{kg}$ ) over $30 \mathrm{~s}$ using a hand-held syringe. To reduce pain on injection, lidocaine (10 mg) was added to each $100 \mathrm{mg}$ of propofol. Once a patient lost consciousness, mask ventilation was gently initiated with the predetermined concentration of sevoflurane in $100 \%$ oxygen, $4 \mathrm{~L}$, to keep the $\mathrm{ETCO}_{2}$ between 25-30 mmHg until intubation was attempted.

Thirty seconds after administration of propofol, remifentanil $(1 \mu \mathrm{g} / \mathrm{kg})$ was infused over $30 \mathrm{~s}$. Remifentanil was made up to a volume of $10 \mathrm{ml}$ in $0.9 \%$ saline. Ninety seconds later, an experienced anesthesiologist unaware of the concentration of sevoflurane employed attempted tracheal intubation by laryngoscope using a Macintosh 3 laryngoscope blade. Each trachea was intubated with a cuffed endotracheal tube $(7.0 \mathrm{~mm}$ for women or $8.0 \mathrm{~mm}$ for men), followed by slow inflation of the cuff over the next $5 \mathrm{~s}$. End-tidal sevoflurane concentration was measured using a Datex anesthesia gas monitor (IntelliVue MP70, Philips, USA) immediately before tracheal intubation. If any difficulty was encountered in performing mask ventilation following induction of anesthesia, or if the intubation was not completed within $30 \mathrm{~s}$, the patient was withdrawn from the study and was given neuromuscular blocking agent for intubation. After intubation, mechanical ventilation with a mixture of oxygen and air was initiated to maintain normocapnia and no further stimulation was applied to the patient.

Intubation conditions were assessed for five variables (jaw relaxation, ease of laryngoscopy, position of the vocal cords, and patient response to intubation and cuff inflation) and scored using a modification of a previous scoring system by another investigator who was blinded to the protocol (Table 1) [15]. Intubation conditions were considered acceptable if all scores were 2 or less and unacceptable if any score was 3 . In both groups, mean arterial blood pressure (MBP), heart rate (HR), and $\mathrm{SpO}_{2}$ were measured prior to induction of anesthesia (T0, pre-induction, baseline), just after propofol (T1) and remifentanil (T2, pre-intubation) infusion, and every minute 
Table 1. Intubation Scores

\begin{tabular}{llll}
\hline & \multicolumn{3}{c}{ Intubation conditions } \\
\cline { 2 - 4 } & \multicolumn{1}{c}{ Score } & Acceptable & Unacceptable \\
\cline { 2 - 4 } & \multicolumn{1}{c}{1} & \multicolumn{1}{c}{3} \\
\hline Jaw relaxation & Relaxed & Acceptable relaxation & Poor relaxation \\
Laryngoscopy & Easy & Fair & Difficult \\
Vocal cords & Abducted & Intermediate & Closed \\
Coughing & None & Diaphragmatic & Severe coughing or bucking \\
Limb movement & None & Slight & Vigorous \\
\hline
\end{tabular}

Excellent: all criteria are score 1, Good: all criteria are either a score 1 or 2, Poor: the presence of a single criterion rated as a score 3. Excellent and good intubation conditions are considered clinically acceptable, whereas poor intubation conditions are not.

Table 2. Subject Demographics

\begin{tabular}{|c|c|c|}
\hline & $\begin{array}{l}\text { Group I } \\
(\mathrm{n}=38)\end{array}$ & $\begin{array}{r}\text { Group II } \\
(\mathrm{n}=38)\end{array}$ \\
\hline Age (yr) & $37.6(16-53)$ & $34.8(17-58)$ \\
\hline Weight (kg) & $56.5 \pm 7.6$ & $57.8 \pm 8.0$ \\
\hline Gender (M/F) & $5 / 33$ & $6 / 32$ \\
\hline $\begin{array}{l}\text { Et sevoflurane } \\
\text { before intubation (vol\%) }\end{array}$ & $1.7 \pm 0.2$ & $2.9 \pm 0.3^{*}$ \\
\hline
\end{tabular}

Values are expressed as mean \pm SD or (range) or number. Et sevoflurane: end-tidal sevoflurane concentration. ${ }^{*} \mathrm{P}<0.05$ compared with the group I.

after tracheal intubation for $3 \mathrm{~min}$ (T3-5).

Patients who could not be intubated within $30 \mathrm{~s}$ during the first attempt and who coughed persistently after tracheal intubation were given an IV dose of rocuronium and no further data were recorded. The occurrence of laryngospasm, bronchospasm, chest rigidity, hypotension, and bradycardia were recorded. Bradycardia was defined as $\mathrm{HR}<50$ beats per min and hypotension was defined as $\mathrm{MBP}<50 \mathrm{mmHg}$.

Prior to initiation of the study, a power analysis was performed. A preliminary study of 15 consecutive patients given $4 \%$ sevoflurane showed acceptable intubation in $60 \%$. Thus, a minimum of 38 patients per group was required to detect an increase from $60 \%$ to $90 \%$ in the proportion of those with acceptable intubation conditions with a power of $80 \%$ and a significance level of $95 \%$.

Data are expressed as means \pm SD or as numbers of patients. Differences between groups were analyzed using an unpaired $t$-test or Mann-Whitney $U$-test. Proportions were analyzed using Chi-square test or Fisher's exact test, as appropriate. Hemodynamic variables within groups were analyzed using Friedman repeated measures analysis of variance on ranks, followed by Student-Newman-Keuls methods. SigmaStat 3.1 (Systat software Inc., Chicago, IL) was used for statistical analysis and $\mathrm{P}<0.05$ was considered to be statistically significant.
Table 3. Number of Patients in Each Group

\begin{tabular}{lcc}
\hline & Group I & Group II \\
\hline $\begin{array}{l}\text { Intubating conditions* } \\
\text { Excellent* }\end{array}$ & 13 & 20 \\
$\quad$ Good & 9 & 15 \\
$\quad$ Poor & 16 & 3 \\
Jaw relaxation & & \\
$\quad$ Score $1 / 2 / 3$ & $35 / 1 / 2$ & $38 / 0 / 0$ \\
Laryngoscopy & & \\
$\quad$ Score $1 / 2 / 3$ & $35 / 1 / 2$ & $38 / 0 / 0$ \\
Vocal cords* & & \\
$\quad$ Score $1 / 2 / 3$ & $24 / 12 / 1$ & $35 / 3 / 0$ \\
Coughing* & & \\
$\quad$ Score $1 / 2 / 3$ & $13 / 10 / 13$ & $20 / 15 / 3$ \\
Limb movements* & & \\
$\quad$ Score $1 / 2 / 3$ & $24 / 10 / 2$ & $36 / 2 / 0$ \\
\hline
\end{tabular}

Values are expressed as number of patients. $* \mathrm{P}<0.05$ compared with the group I.

\section{Results}

A total of 76 patients were enrolled in this study. Two patients in each group were withdrawn because of unexpectedly difficult intubation or no visualization of vocal cords, and replaced by an additional 4 patients. There were no significant differences between the two groups in terms of age, weight, and gender ratios (Table 2). The mean end-tidal sevoflurane concentration just before intubation was significantly higher in group II (2.9\%) than in group I $(1.7 \%)(\mathrm{P}<0.001)$.

The overall incidence of clinically acceptable intubation conditions was significantly higher in group II (92\%) than group I (58\%) $(\mathrm{P}=0.001)$ (Table 3). Excellent intubation conditions (score of 1 in all categories) were achieved more often in group II (20/38 patients, 53\%) than those in group I (13/38 patients, $34 \%)$. Although there was no failed intubation in group II, tracheal intubation failed in two patients of group I because of poor jaw relaxation or closed vocal cords. Three patients in the group I required rocuronium because of failed intubation and persistent coughing. Scores for vocal cord position, coughing, 


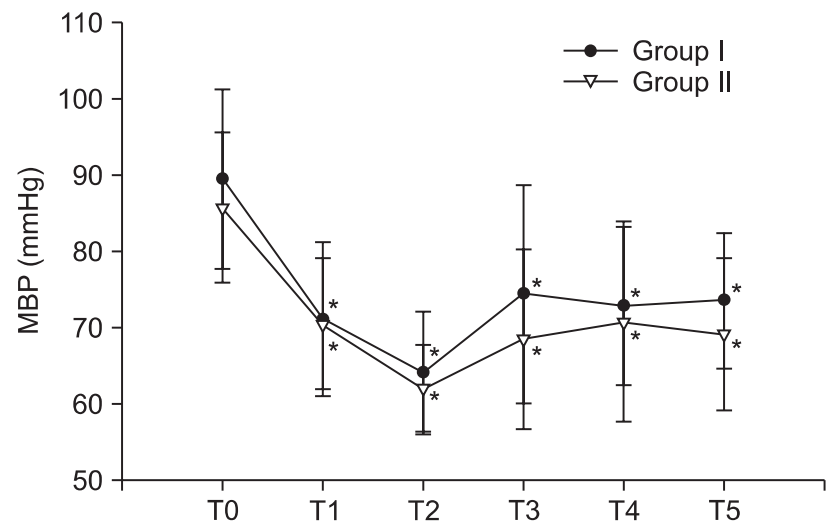

Fig. 1. Changes in mean blood pressure during the study. Data are presented as mean \pm SD. T0: before induction, T1: after propofol infusion, T2: after remifentanil infusion, T3-5: every $1 \mathrm{~min}$ after intubation for $3 \mathrm{~min}$. ${ }^{*} \mathrm{P}<0.05$ when compared with $\mathrm{T} 0$.

and limb movement were significantly lower in group II than those in group I (Table 3$)(\mathrm{P}<0.05)$, but not scores for jaw relaxation or ease of laryngoscope $(\mathrm{P}>0.05)$.

Before induction, pre-induction MBP and HR were not significantly different between groups $(\mathrm{P}>0.05)$ (Fig. 1 and 2). MBP remained significantly lower than the pre-induction level in both groups $(\mathrm{P}<0.001)$. HR was unchanged or lower compared with pre-induction levels in both groups, but MBP and HR were not different at any time $(\mathrm{P}>0.05)$. There was no episode of opioid-induced muscle rigidity, bradycardia, or hypotension requiring treatment and no other side effects were seen. $\mathrm{SpO}_{2}$ remained at the pre-induction levels, $97-100 \%$, in all patients.

\section{Discussion}

We have shown here that the addition of $7 \%$ sevoflurane to propofol $(2 \mathrm{mg} / \mathrm{kg})$ and remifentanil $(1 \mu \mathrm{g} / \mathrm{kg})$ reliably provided acceptable conditions for IWNA. The addition of $7 \%$ sevoflurane produced significantly better intubation conditions than $4 \%$ sevoflurane and was not associated with any incidence of failed intubation. In addition, the position of the vocal cords was more favorable and the degree of coughing and limb movements following intubation lower in patients given $7 \%$ sevoflurane, probably due to a deeper level of anesthesia.

The use of sevoflurane for induction and tracheal intubation has been widely investigated in children and adults. However, the end-tidal sevoflurane concentration required for tracheal intubation was high $\left(\mathrm{ED}_{95}=8.07 \%\right)$ and the time required to reach an adequate depth of anesthesia in adults using sevoflurane alone was over 6 min $[12,16]$. A bolus or continuous infusion of remifentanil after sevoflurane induction facilitated IWNA [11-14]. Joo et al. [11] showed that the addition of remifentanil $(2 \mu \mathrm{g} / \mathrm{kg})$ after induction with $8 \%$ sevoflurane and $50 \%$ nitrous oxide provided optimal intubation conditions

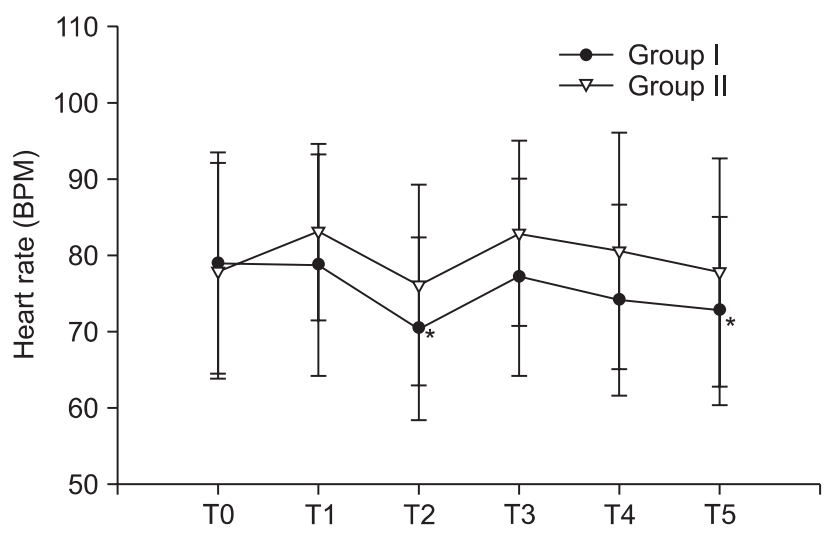

Fig. 2. Changes in heart rate during the study. Data are presented as mean \pm SD. T0: before induction, T1: after propofol infusion, T2: after remifentanil infusion, T3-5: every $1 \mathrm{~min}$ after intubation for 3 min. ${ }^{*} \mathrm{P}<0.05$ when compared with $\mathrm{T} 0$.

in all patients within 3 min. However, the combined use of sevoflurane with high-dose remifentanil was associated with hypotension in $29 \%$ of patients.

The beneficial effects of our method include, in the majority of patients, acceptable intubation conditions without neuromuscular blocking agents, within $3 \mathrm{~min}$, and without hemodynamic compromise. The high incidence of acceptable intubation conditions was comparable with that seen in the $60-100 \%$ of patients treated by propofol/remifentanil tracheal intubation without neuromuscular blocking agents [5-10]. These earlier studies, however, used larger doses of propofol or remifentanil to obtain optimal intubation conditions, with occasional development of hypotension [8-10]. The addition of sevoflurane at the induction of anesthesia enabled the use of lower doses propofol and remifentanil to achieve comparable results. We selected a propofol dose of $2 \mathrm{mg} / \mathrm{kg}$ because this dose is frequently recommended for anesthesia induction in adults. The remifentanil dose of $2-5 \mu \mathrm{g} / \mathrm{kg}$ was employed because it produces adequate conditions for IWNA when combined with propofol $(2 \mathrm{mg} / \mathrm{kg})$ in pre-medicated healthy adults [6-10]. As remifentanil may cause muscle rigidity in proportion to dose and rate of administration [17], we choose a lower dose of remifentanil $(1 \mu \mathrm{g} / \mathrm{kg})$ and infused the drug over $30 \mathrm{~s}$.

The timing of drug administration was crucial to the high success rate of IWNA achieved in this report. When the peak site effects of propofol and remifentanil occur simultaneously, optimal intubation conditions are attained [18]. The peak effect of remifentanil occurred approximately $90 \mathrm{~s}$ after a bolus dose [19], and the propofol effect peaks $180 \mathrm{~s}$ after administration [20]. We therefore timed the administration of these drugs to synchronize peak site effects at the time of intubation, and hypothesized that the addition of sevoflurane between induction of anesthesia and intubation would provide better intubation conditions. 
The balanced use of propofol, remifentanil, and sevoflurane made it possible to avoid hemodymamic depression, which has been noted when large doses of one or two agents [8-11]. MBP was lower at all time points than the pre-induction level, but no patient developed hypotension requiring treatment. Glycopyrrolate was administrated as an anti-sialogue and as prophylaxis against bradycardia, and clinically significant bradycardia was not encountered. As our method attenuated the hemodynamic response to tracheal intubation in all patients, the approach may be useful when prevention of cardiovascular intubation response is required, without the use of large doses of opioids or adjunctive cardiovascular depressant agents.

Our study has several limitations. First, it is important to consider patient demographic characteristics, such as gender and age, prior to use of this method. Gender differences can affect the pharmacodynamics and pharmacokinetics of propofol [21], and women have significantly higher propofol serum concentrations at the point of loss of consciousness [22]. In addition, older patients are more sensitive to opioids than younger ones. Our study population showed a predominance of young females, which should be considered when interpreting our data. Second, the decreases in MBP and HR were well tolerated by the healthy, well-hydrated patients of our study. However, this method cannot be recommended for hypovolemic or debilitated patients. Another limitation was the failure to include a control group (receiving only propofol and remifentanil without sevoflurane) in this study design. In a small pilot study, the IWNA with propofol $(2 \mathrm{mg} / \mathrm{kg})$ and remifentanil $(1 \mu \mathrm{g} / \mathrm{kg})$ failed in four successive patients. Thus, for ethical reasons, we could not include a control group.

In conclusion, our results suggest that the addition of $7 \%$ sevoflurane to timely injections of low dose propofol (2 mg/ $\mathrm{kg}$ ) and remifentanil $(1 \mu \mathrm{g} / \mathrm{kg})$ provides satisfactory intubation conditions without hemodynamic compromise in healthy premedicated patients with favorable airway anatomy. This method may be appropriate when tracheal intubation is necessary but neuromuscular blockade is not desired.

\section{References}

1. Taha S, Siddik-Sayyid S, Alameddine M, Wakim C, Dahabra C, Moussa A, et al. Propofol is superior to thiopental for intubation without muscle relaxants. Can J Anaesth 2005; 52: 249-53.

2. McKeating K, Bali IM, Dundee JW. The effects of thiopentone and propofol on upper airway integrity. Anaesthesia 1988; 43: 638-40.

3. Scanlon P, Carey M, Power M, Kirby F. Patient response to laryngeal mask insertion after induction of anaesthesia with propofol or thiopentone. Can J Anaesth 1993; 40: 816-8.

4. Erhan E, Ugur G, Gunusen I, Alper I, Ozyar B. Propofol - not thiopental or etomidate - with remifentanil provides adequate intubating con- ditions in the absence of neuromuscular blockade. Can J Anaesth 2003; 50: 108-15.

5. Klemola UM, Hiller A. Tracheal intubation after induction of anesthesia in children with propofol--remifentanil or propofolrocuronium. Can J Anaesth 2000; 47: 854-9.

6. Stevens JB, Wheatley L. Tracheal intubation in ambulatory surgery patients: using remifentanil and propofol without muscle relaxants. Anesth Analg 1998; 86: 45-9.

7. Erhan E, Ugur G, Alper I, Gunusen I, Ozyar B. Tracheal intubation without muscle relaxants: remifentanil or alfentanil in combination with propofol. Eur J Anaesthesiol 2003; 20: 37-43.

8. Grant S, Noble S, Woods A, Murdoch J, Davidson A. Assessment of intubating conditions in adults after induction with propofol and varying doses of remifentanil. Br J Anaesth 1998; 81: 540-3.

9. Alexander R, Olufolabi AJ, Booth J, El-Moalem HE, Glass PS. Dosing study of remifentanil and propofol for tracheal intubation without the use of muscle relaxants. Anaesthesia 1999; 54: 1037-40.

10. Woods AW, Grant S, Harten J, Noble JS, Davidson JA. Tracheal intubating conditions after induction with propofol, remifentanil and lignocaine. Eur J Anaesthesiol 1998; 15: 714-8.

11. Joo HS, Perks WJ, Belo SE. Sevoflurane with remifentanil allows rapid tracheal intubation without neuromuscular blocking agents. Can J Anaesth 2001; 48: 646-50.

12. Muzi M, Robinson BJ, Ebert TJ, O'Brien TJ. Induction of anesthesia and tracheal intubation with sevoflurane in adults. Anesthesiology 1996; 85: 536-43.

13. Cros AM, Lopez C, Kandel T, Sztark F. Determination of sevoflurane alveolar concentration for tracheal intubation with remifentanil, and no muscle relaxant. Anaesthesia 2000; 55: 965-9.

14. Sztark F, Chopin F, Bonnet A, Cros AM. Concentration of remifentanil needed for tracheal intubation with sevoflurane at $1 \mathrm{MAC}$ in adult patients. Eur J Anaesthesiol 2005; 22: 919-24.

15. Sluga M, Ummenhofer W, Studer W, Siegemund M, Marsch SC. Rocuronium versus succinylcholine for rapid sequence induction of anesthesia and endotracheal intubation: a prospective, randomized trial in emergent cases. Anesth Analg 2005; 101: 1356-61.

16. Kimura T, Watanabe S, Asakura N, Inomata S, Okada M, Taguchi M. Determination of end-tidal sevoflurane concentration for tracheal intubation and minimum alveolar anesthetic concentration in adults. Anesth Analg 1994; 79: 378-81.

17. Thompson JP, Rowbotham DJ. Remifentanil:an opioid for the 21st century. Br J Anaesth 1996; 76: 341-3.

18. Trabold F, Casetta M, Duranteau J, Albaladejo P, Mazoit JX, Samii $\mathrm{K}$, et al. Propofol and remifentanil for intubation without muscle relaxant: the effect of the order of injection. Acta Anaesthesiol Scand 2004; 48: 35-9.

19. Glass PS, Gan TJ, Howell S. A review of the pharmacokinetics and pharmacodynamics of remifentanil. Anesth Analg 1999; 89: S7-14.

20. Gepts E. Pharmacokinetic concepts for TCI anaesthesia. Anaesthesia 1998; 53 Suppl 1: 4-12.

21. Kodaka M, Johansen JW, Sebel PS. The influence of gender on loss of consciousness with sevoflurane or propofol. Anesth Analg 2005; 101: 377-81.

22. Kodaka M, Suzuki T, Maeyama A, Koyama K, Miyao H. Gender differences between predicted and measured propofol CP50 for loss of consciousness. J Clin Anesth 2006; 18: 486-9. 